\title{
The cost effectiveness of elective laparoscopic sigmoid resection for symptomatic diverticular disease: financial outcome of the randomized control Sigma trial
}

\author{
Bastiaan R. Klarenbeek - Veerle M. H. Coupé • \\ Donald L. van der Peet $\cdot$ Miguel A. Cuesta
}

Received: 8 February 2010/ Accepted: 8 July 2010/Published online: 27 July 2010

(C) Springer Science+Business Media, LLC 2010

\begin{abstract}
Background Direct healthcare costs of patients with symptomatic diverticular disease randomized for either laparoscopic or open elective sigmoid resection are compared. Cost-effectiveness analysis of the laparoscopic approach compared with open sigmoid resections is presented.

Methods An economic evaluation of the randomized control Sigma trial was conducted, comparing elective laparoscopic sigmoid resection (LSR) to open sigmoid resection (OSR) in patients with symptomatic diverticulitis. Prospective registration of detailed intervention units per patient resulted in actual resource use per individual patient. To avoid distributional assumptions, the nonparametric bootstrap was applied. For the cost-effectiveness analysis, differences in total cost between LSR and OSR were compared with the differences in VAS pain score, SF36 values for general health, and complication rate.

Results The difference in total healthcare costs between the group that received LSR ( $€$ 9969) and the group that received OSR ( $€ 9366)$ was not statistically significant. The slight increase in total costs was determined mainly by the significantly higher operation costs of LSR ( $€ 6663$ vs. $€ 5306$ ). Lower costs for hospitalization ( $€ 2983$ vs. $€ 3598$ ), blood products ( $€ 87$ vs. $€ 240$ ), paramedical services $(€ 157$ vs. $€ 278$ ), and emergency attendance ( $€ 72$ vs. $€ 115$ ) in the LSR group partially compensated these increased operation costs. The incremental cost-effectiveness ratios (ICER)
\end{abstract}

B. R. Klarenbeek $(\bowtie)$ D. L. van der Peet · M. A. Cuesta Department of Surgery, VU University Medical Center,

Amsterdam, The Netherlands

e-mail: br.klarenbeek@vumc.nl

V. M. H. Coupé

Department of Epidemiology and Biostatistics, VU University

Medical Center, Amsterdam, The Netherlands indicate that improvements in pain, quality of life, and complication rate could be achieved at limited costs.

Conclusion Total healthcare costs of laparoscopic and open elective sigmoid resections for symptomatic diverticular disease are similar. As the clinical outcomes are in favor of the LSR group, candidates for an elective sigmoid resection should preferably be approached laparoscopically.

Keywords Diverticulitis - Diverticular disease · Laparoscopy $\cdot$ Cost analysis

Diverticular disease is one of the major gastrointestinal disorders in the Western society, with an incidence rate of $33 \%$ in the population over 45 years of age and $66 \%$ in the population older than 85 years of age [1]. Approximately $10-25 \%$ of patients with asymptomatic diverticulosis will develop acute diverticulitis [2]. Each year diverticular disease accounts for 312,000 hospital admissions in the United States, costing a total of $\$ 2,667,000,000[3,4]$.

The treatment of diverticular disease depends on the severity of the disease, varying from light symptomatic diverticulosis to perforated diverticulitis [5]. An elective sigmoid resection is indicated in complicated cases such as stricture of the sigmoid colon, a fistula to a hollow organ, or recurrent diverticular bleeding $[6,7]$. Other indications like recurrent episodes or persistent complaints in young patients and certain high-risk groups (i.e., immunocompromised or users of NSAIDs) remain controversial [8, 9].

Over the past two decades the laparoscopic approach in the management of diverticular disease has gained acceptance. Several nonrandomized comparison studies showed beneficial outcomes of laparoscopic sigmoid resection, such as shorter hospitalization and a reduction in postoperative complication rates [10-12]. The reported Sigma 
trial was the first randomized control trial to compare laparoscopic versus open elective sigmoid resections in patients with symptomatic diverticular disease [13]. The short-term results showed that the laparoscopic approach was associated with a significant $15.4 \%$ reduction in major complication rate, less pain, shorter hospitalization, and improved quality of life at the cost of a longer operating time [14]. Despite these short-term benefits, comparable outcomes were found at 6 months follow-up in terms of late complications, mortality, and quality of life.

In contrast, concerns remain regarding the higher healthcare costs associated with laparoscopic colorectal surgery because of the longer operative procedures and the greater use of disposable products. Studies comparing the costs of laparoscopic and open colorectal surgery for various benign or malignant indications report conflicting results [15]. When diverticular disease is considered, only three nonrandomized cohort studies could be identified [16-18]. In this article, the direct healthcare costs of patients with symptomatic diverticular disease randomized for either laparoscopic or open elective sigmoid resection are compared. Furthermore, a cost-effectiveness analysis of the laparoscopic approach compared with open sigmoid resections is presented.

\section{Methods}

\section{Study design}

An economic evaluation of the Sigma trial was conducted comparing elective laparoscopic sigmoid resection (LSR) to open sigmoid resection (OSR) in patients with symptomatic diverticulitis of the sigmoid colon. The Sigma trial was a prospective, multicenter, double-blind, parallel-arm, randomized control study. Eligible patients were randomized to either LSR or OSR. The cost effectiveness was investigated for patients included in the VU University Medical Center.

End points included postoperative mortality, and complications were classified as minor, major, and late. Secondary outcome measures included operating time, estimated blood loss, conversion rate, hospital stay, usage of analgesia, Visual Analog Scale (VAS) pain score, resumption of diet, and quality of life assessment by the Short Form-36 (SF-36) questionnaire.

Written informed consent was obtained from eligible patients. The generator of the allocation sequence was separated from the executor. Blinding was ensured by using an opaque wound dressing to cover the abdomen.

Symptomatic diverticulitis of the sigmoid colon was defined as recurrent disease Hinchey I, IIa, IIb, symptomatic stricture, or severe rectal bleeding. The decision to discharge patients was made by independent physicians blind to the allocation sequence. Patients visited the outpatient clinic 6 weeks and 6 months after surgery.

The study was conducted in accordance to the principles of the Declaration of Helsinki and "good clinical practice" guidelines. The study protocol was approved by the Institutional Review Boards of all participating institutions. Data were analyzed according to the intention-to-treat principle. Methodological and operative details can be found in the published protocol [13]. Study end points, secondary outcome measures, and their assessment are described in the clinical paper [14].

\section{Economic evaluation}

The economic evaluation was conducted from a hospital perspective. As such, only direct healthcare costs were considered. The study was carried out from February 2002 to December 2006. Therefore, all costs were converted to fiscal year 2005 euros.

Since no uniformity or transparency exists for cost registration in the different participating clinics and countries, only patients who were treated in the VU University Medical Center were included. Data on resource use were aggregated from the TOREN software program (iSOFT ${ }^{\circledR}$, Leiden, The Netherlands). This program enables prospective registration of detailed intervention units per patient, such as materials (sutures and disposables), medical equipment, personnel costs, and specialist fees. Parameters such as operations, admission days, or diagnostic tools are composed of several intervention units resulting in actual resource use per individual patient. Different parameters are grouped into nine of categories as shown in Table 1.

The translation of resource utilization into costs was provided by the TRAG PI software program (TRAG Performance Intelligence ${ }^{\circledR}$, Zeist, The Netherlands). This software program provided information on direct cost per intervention unit per patient extracted from the prospective TOREN registry. These cost prices reflect costs of real resource use and include overhead costs.

Actual costs per individual patient are presented in eight categories, as well as total direct costs for the period of 30 days after surgery. For the 6-months follow-up period, only total healthcare costs are provided.

Statistical analysis

The mean differences in costs between LSR and OSR were computed and the $95 \%$ confidence interval (95\% CI) for this difference was calculated. Because cost data are typically skewed, confidence intervals for cost differences cannot be estimated using conventional methods that assume normality. To avoid distributional assumptions, the 
Table 1 Resources per category extracted from the TRAG PI software program

\begin{tabular}{ll}
\hline Category & Recourse examples \\
\hline Hospitalization & Admission days, laboratory testing, medication, nurses' fee \\
Operation & $\begin{array}{c}\text { Operating room per hour, sterilization costs, disposable } \\
\text { materials, specialists' fee }\end{array}$ \\
Imaging & CT scan, ultrasound, radiograph, barium enema \\
Diagnostic procedures & Colonoscopy, EKG \\
Blood products & Packed cells, fresh frozen plasma \\
Consulting specialists & Cardiology, Internal Medicine, Dermatology \\
Paramedical services & Physiotherapy, dietician, stoma care, social worker \\
Emergency and outpatient attendance & Follow-up and/or emergency visits for complications \\
\hline
\end{tabular}

nonparametric bootstrap was applied [19, 20]. Basically, in the nonparametric bootstrap, samples of the same size as the original data set are drawn by sampling with replacement from the observed data. These bootstrap samples can be used to estimate standard errors and confidence intervals. To obtain $95 \%$ confidence intervals for cost differences, a nonparametric bootstrap with 2000 replications was performed [21].

For the cost-effectiveness analysis, differences in total cost between LSR and OSR were compared with the differences in VAS pain scores and SF-36 values for general health. Furthermore, the difference in total costs was compared to the difference in complication rate, as this was one of the primary end points of the study. Uncertainty around the cost-effectiveness ratios was estimated using the bias-corrected and accelerated bootstrapping method (5000 replications) and presented on a cost-effectiveness plane [21, 22].

A sensitivity analysis was carried out to study the effect of excluding the extreme costs incurred by one patient who had a tenfold increase in costs of hospitalization due to a severely complicated course at 6 months follow-up.

\section{Results}

Clinical outcomes

At baseline, no differences in clinical characteristics were found between LSR and OSR. Short-term analysis showed that LSR was associated with a $15.4 \%$ reduction in major complication rate, less pain, shorter hospitalization, and improved quality of life at the cost of longer operating time. Full details on the clinical outcomes are presented in the clinical papers $[13,14]$.

\section{Resource use}

A total of 104 patients were included in the trial, of which 57 patients were treated at the VU University Medical Center. Twenty-seven were randomized to the LSR group and 30 to the OSR group. Table 2 lists a selection of the most common healthcare resource items. Note that infrequently used resource items are not displayed but do contribute to total costs.

LSR was associated with less hospitalization, paramedical services, and emergency attendance, but increased use of disposable materials, imaging modalities, and operative time. Except for operative time, differences were small and not statistically significant.

Table 2 Mean (SD) resource utilization per treatment group within 30 days

\begin{tabular}{|c|c|c|c|c|}
\hline & LSR & $n$ & OSR & $n$ \\
\hline Hospital stay (days) & $8.41(4.81)$ & 27 & $9.30(4.66)$ & 30 \\
\hline Operative time $(\min )^{*}$ & $211.73(33.52)$ & 27 & $145.59(40.87)$ & 30 \\
\hline \multicolumn{5}{|l|}{ Imaging } \\
\hline CT abdomen & $0.22(0.97)$ & 3 & $0.07(0.25)$ & 2 \\
\hline Abdominal ultrasound & $0.07(0.27)$ & 2 & 0 & 0 \\
\hline Chest or abdominal X-ray & $0.67(1.07)$ & 11 & $0.70(1.06)$ & 15 \\
\hline Barium enema & $0.07(0.27)$ & 2 & $0.07(0.25)$ & 2 \\
\hline \multicolumn{5}{|l|}{ Diagnostic procedures } \\
\hline Colonoscopy & 0 & 0 & $0.10(0.55)$ & 1 \\
\hline EKG & $0.41(0.69)$ & 9 & $0.27(0.58)$ & 6 \\
\hline \multicolumn{5}{|l|}{ Laboratory } \\
\hline Laboratory tests & $11.44(14.38)$ & 24 & $11.23(14.63)$ & 29 \\
\hline Blood products & $0.37(0.97)$ & 4 & $1.23(2.71)$ & 8 \\
\hline \multicolumn{5}{|l|}{ Consulting specialists (visits) } \\
\hline Internal Medicine & $0.15(0.46)$ & 3 & $0.13(0.35)$ & 4 \\
\hline Cardiology & $0.11(0.32)$ & 3 & $0.10(0.31)$ & 3 \\
\hline \multicolumn{5}{|l|}{ Paramedical services (visits) } \\
\hline Physiotherapy & $0.56(1.28)$ & 8 & $2.20(3.86)$ & 10 \\
\hline Dietician & $0.44(1.31)$ & 4 & $0.57(2.75)$ & 2 \\
\hline Stoma-care & $1.00(2.92)$ & 3 & $1.77(3.57)$ & 7 \\
\hline \multicolumn{5}{|c|}{ Emergency and outpatient attendance (visits) } \\
\hline Follow-up & $1.56(0.75)$ & 27 & $1.33(1.09)$ & 23 \\
\hline Emergency & $0.07(0.27)$ & 2 & $0.13(0.43)$ & 3 \\
\hline
\end{tabular}

$n$ columns indicate the number of patients with resource use

$* p=0.000$; no other significant differences 


\section{Costs}

Table 3 shows the mean costs for the two groups per category. Total healthcare costs within 30 days were $€ 9969$ for LSR and $€ 9366$ for OSR. Compared with OSR, this was a $6.4 \%$ ( $€ 603)$ increase in total healthcare costs within 30 days for LSR, which was not statistically significant $(95 \%$ $\mathrm{CI}=-957$ to 2162 ). These costs were determined mainly by the significantly higher operation cost of $€ 6663$ for LSR vs. $€ 5306$ for OSR. These increased costs were partially compensated for by lower costs for hospitalization (LSR $€ 2983$ vs. OSR $€ 3598$ ), blood products (LSR $€ 87$ vs. OSR $€ 240$ ), paramedical services (LSR $€ 157$ vs. OSR $€ 278$ ), and emergency attendance (LSR $€ 72$ vs. OSR $€ 115$ ). These latter differences, however, were not significant.

Total costs at 6 months follow-up were $€ 17,203$ for LSR and $€ 11,357$ for OSR (difference $=€ 5846$; $95 \%$ $\mathrm{CI}=-6105$ to 17,797 ) (see Table 4). A substantial increase in total costs at 6 months follow-up was found in the LSR group, which was solely determined by a very complicated course for one patient. This patient underwent a Hartmann's procedure for a large intra-abdominal abscess, followed by anastomotic leakage when restoration of bowel continuity was attempted. Major contributors to the enormous costs were prolonged stay in the intensive care unit, blood products, a total of nine reoperations and 23 CT scans. When excluding this single patient in a sensitivity analysis, total costs at 6 months were $€ 11,344$ for LSR and $€ 11,357$ for OSR (difference $=€ 12 ; 95 \%$ $\mathrm{CI}=-3438$ to 3499 ).

Cost effectiveness

Table 4 shows the total costs and effects within 30 days and at 6 months follow-up for the different outcome measures. Table 5 shows the differences in total costs and
Table 3 Mean (SD) costs ( $€$ ) per treatment group within 30 days

${ }^{\text {a }}$ Difference with $95 \%$ CI obtained from a nonparametric bootstrap method with 2000 replications

Table 4 Mean costs $(€)$ and effects by treatment group for all patients

$* p$ values for effect differences

Table 5 Mean cost $(€)$ and effect differences between treatment groups and costeffectiveness ratios for all patients

a A negative sign indicates a decrease in pain and complications, meaning actual improvement in effect

\begin{tabular}{|c|c|c|c|}
\hline & $\operatorname{LSR}(n=27)$ & OSR $(n=30)$ & Difference $(95 \% \mathrm{CI})^{\mathrm{a}}$ \\
\hline Hospitalization & $2982.80(1403.45)$ & $3597.71(1850.73)$ & $-614.69(-1500.83$ to 248.17$)$ \\
\hline Operation & $6662.80(1402.67)$ & $5306.21(1480.59)$ & 1356.57 (319.18 to 2156.52$)$ \\
\hline Imaging & $100.04(186.67)$ & $86.39(140.93)$ & $13.66(-79.98$ to 94.17$)$ \\
\hline Diagnostic procedures & $62.19(128.97)$ & $53.50(128.16)$ & $8.69(-62.23$ to 72.64$)$ \\
\hline Blood products & $87.24(198.78)$ & $240.08(528.86)$ & $-152.88(-305.24$ to 42.73$)$ \\
\hline Consulting specialists & $63.01(176.97)$ & $47.69(110.95)$ & $15.32(-70.69$ to 84.29$)$ \\
\hline Paramedical services & $157.32(426.52)$ & $278.39(568.60)$ & $-121.07(-277.56$ to 150,72$)$ \\
\hline $\begin{array}{l}\text { Emergency and } \\
\text { outpatient attendance }\end{array}$ & $71.89(80.27)$ & $115.26(135.55)$ & $-43.38(-98.53$ to 10.71$)$ \\
\hline Total costs & $9969.44(2578.00)$ & $9366.03(3127.18)$ & $603.37(-956.86$ to 2161.77$)$ \\
\hline
\end{tabular}

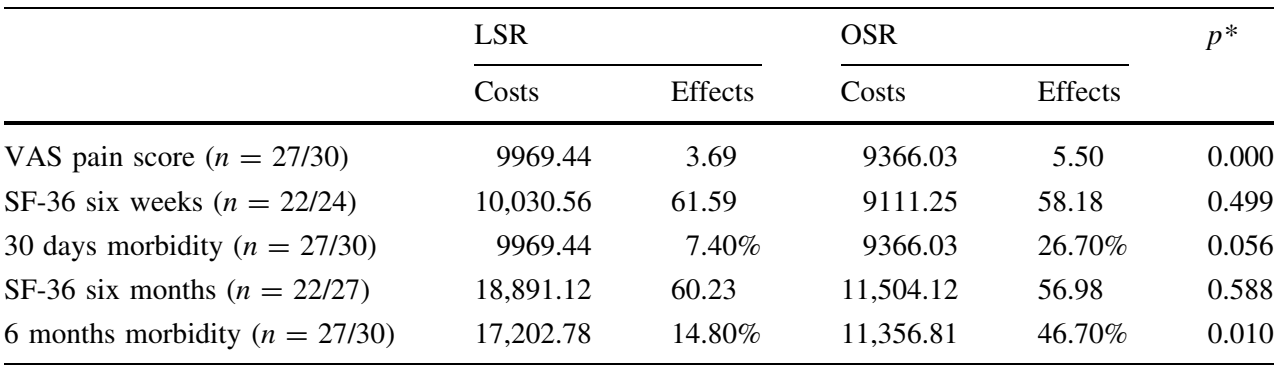

\begin{tabular}{|c|c|c|c|}
\hline & \multicolumn{3}{|l|}{ LSR-OSR } \\
\hline & $\begin{array}{l}\text { Cost } \\
\text { difference }\end{array}$ & $\begin{array}{l}\text { Effect } \\
\text { difference }\end{array}$ & ICER $(95 \% \mathrm{CI})$ \\
\hline VAS pain score $(n=27 / 30)$ & 603.41 & $-1.82^{\mathrm{a}}$ & $332.46(-302.30$ to 1553.26$)$ \\
\hline SF-36 six weeks $(n=22 / 24)$ & 919.30 & 3.41 & $269.29(-1905.13$ to 2276.33$)$ \\
\hline 30 days morbidity $(n=27 / 30)$ & 603.41 & $-19.30 \%^{\mathrm{a}}$ & $31.26(-458.32$ to 680.32$)$ \\
\hline SF-36 six months $(n=22 / 27)$ & 7387.00 & 3.25 & $2271.53(-21957.08$ to 23502.55$)$ \\
\hline 6 months morbidity $(n=27 / 30)$ & 5845.97 & $-31.90 \%^{\mathrm{a}}$ & $183.26(-59.38$ to 1303.27$)$ \\
\hline
\end{tabular}


effects, the incremental cost-effectiveness ratios (ICER), and their $95 \%$ confidence intervals $(95 \% \mathrm{CI})$.

The ICER for pain was $€ 332$ per unit on the Visual Analog Scale (VAS). Considering that a negative VAS difference is an improvement, this incremental cost-effectiveness ratio means that LSR costs $€ 332$ per VAS unit that is gained by performing LSR instead of OSR. Figure $1 \mathrm{~A}$ shows the cost-effectiveness plane for improvement in VAS. In total, $89 \%$ of the cost-effect pairs lie above the $X$ axis and right to the $Y$ axis, the area where LSR is associated with decreasing pain but more expenses.

The cost-effectiveness ratio for the general health score of the Short Form-36 (SF-36) at 6 weeks was $€ 269$ per SF36 unit, meaning that LSR costs $€ 269$ per gained general health unit. Figure 1B shows the corresponding costeffectiveness plane. Seventy-four percent of the cost-effect pairs lie in the upper-right quadrant, corresponding with increased quality of life and higher costs.

Complication rate within 30 days of surgery was associated with a cost-effectiveness ratio of $€ 31$, implicating a cost of $€ 31$ per percent decrease in complication rate. Figure 1C shows the corresponding cost-effectiveness plane. For $85 \%$ of the cost-effect pairs, the complication rate was decreased at a higher cost.

The ICERs for general health score and complication rate at 6 months follow-up are shown in Table 5. The large 95\% confidence intervals illustrate the influence of the single extremely complicated patient. To study the effect of this outlier, a sensitivity analysis was carried out. The exclusion of the extreme costs incurred by one severely complicated patient resulted in an ICER for general health of $€ 57$ (95\% CI $=-2753$ to 3436) and for complication rate of $-€ 0.35$ (95\% CI $=-73$ to 140$)$. Although slightly higher cost-effectiveness ratios were found for LSR at six months follow-up (not significant), similar values for LSR and OSR were found in this sensitivity analysis.

\section{Discussion}

In this study we investigated the cost effectiveness of laparoscopic (LSR) versus open elective sigmoid resection (OSR) for patients with symptomatic diverticular disease. The difference in total healthcare costs between the LSR group and the OSR group was not statistically significant. Overall, costs for the LSR group were somewhat higher than for the OSR group ( $€ 603)$, but this difference was surrounded by large confidence bounds ( -957 to 2162). The increase in total costs was determined mainly by the significantly higher operation costs of the laparoscopic approach. Lower costs for hospitalization, blood products, paramedical services, and emergency attendance in the LSR group partially compensated these increased operation costs. The effect differences between the two treatment groups, in particular, during the first 30 days, were in favor of LSR, with a significant decrease in pain (VAS) and complication rate and a significant increase in quality of life (SF-36).

The cost-effectiveness analyses showed that these improvements in health could be achieved at limited costs (e.g., $€ 31$ per percentage decrease in major complication within 30 days). As was observed for the costs, the incremental cost-effectiveness ratios of LSR versus OSR were also surrounded by large confidence bounds, including negative ICERs indicating that there is even a small chance that the health gain can be achieved at lower costs. Based on these findings it can be concluded that laparoscopic and open sigmoid resection are equivalent treatment options with respect to costs and that a choice should thus be based on the observed effect differences only.

An interesting finding regarding complication rates resulted from the subgroup analysis of the VU University Medical Center population. In the clinical paper on shortterm results of the Sigma trial, a significant $15.4 \%$ reduction in major morbidity rate for the laparoscopic approach was presented; when analyzing those $57 \mathrm{VU}$ patients, a $19.3 \%$ reduction was observed $(p=0.056)$. Unpublished data on 6 months follow-up demonstrate late complication rates of 13.5 and $23.1 \%$ for LSR and OSR, respectively $(p=0.205)$. The pooling of both short-term and late complications per patient included in the VU University Medical Center resulted in a significant $31.9 \%$ reduction of total complication rates at 6 months follow-up (LSR 14.8\% vs. OSR $46.7 \%$; $p=0.010$ ).

The main strength of this study lies in the cost-registration method. In most studies an estimation of mean costs of interventions is made based on average resource use [15]. In this study the resource utilization results from prospective registration of detailed resource items per patient. This registry therefore enables a more accurate calculation of actual costs per individual patient.

In addition, this study is the first to relate total healthcare costs to clinical outcome measures of laparoscopic versus open resections in colorectal surgery. Several papers presented effects and costs side by side, but all did not conduct cost-effectiveness analyses. Beneficial outcomes after laparoscopic sigmoid resections for diverticular disease, such as shorter hospitalization and a reduction in postoperative complication rates, have been reported in nonrandomized comparison studies [10-12]. In recent literature only three papers report on a comparison of costs between laparoscopic and open sigmoid resections for diverticular disease [16-18]. Although there are differences in the assessment of resource utilization, in calculations of total costs, and in use of statistical methods, all three studies demonstrate a decrease in total hospital costs of 23-25\%. Limited 
Fig. 1 Cost-effectiveness planes. A Cost-effectiveness plane for improvement in VAS pain score for LSR vs. OSR. B Cost-effectiveness plane for improvement in general health score of the SF-36 for LSR vs. OSR. C Cost-effectiveness plane for reduction in major complication rate for LSR versus OSR
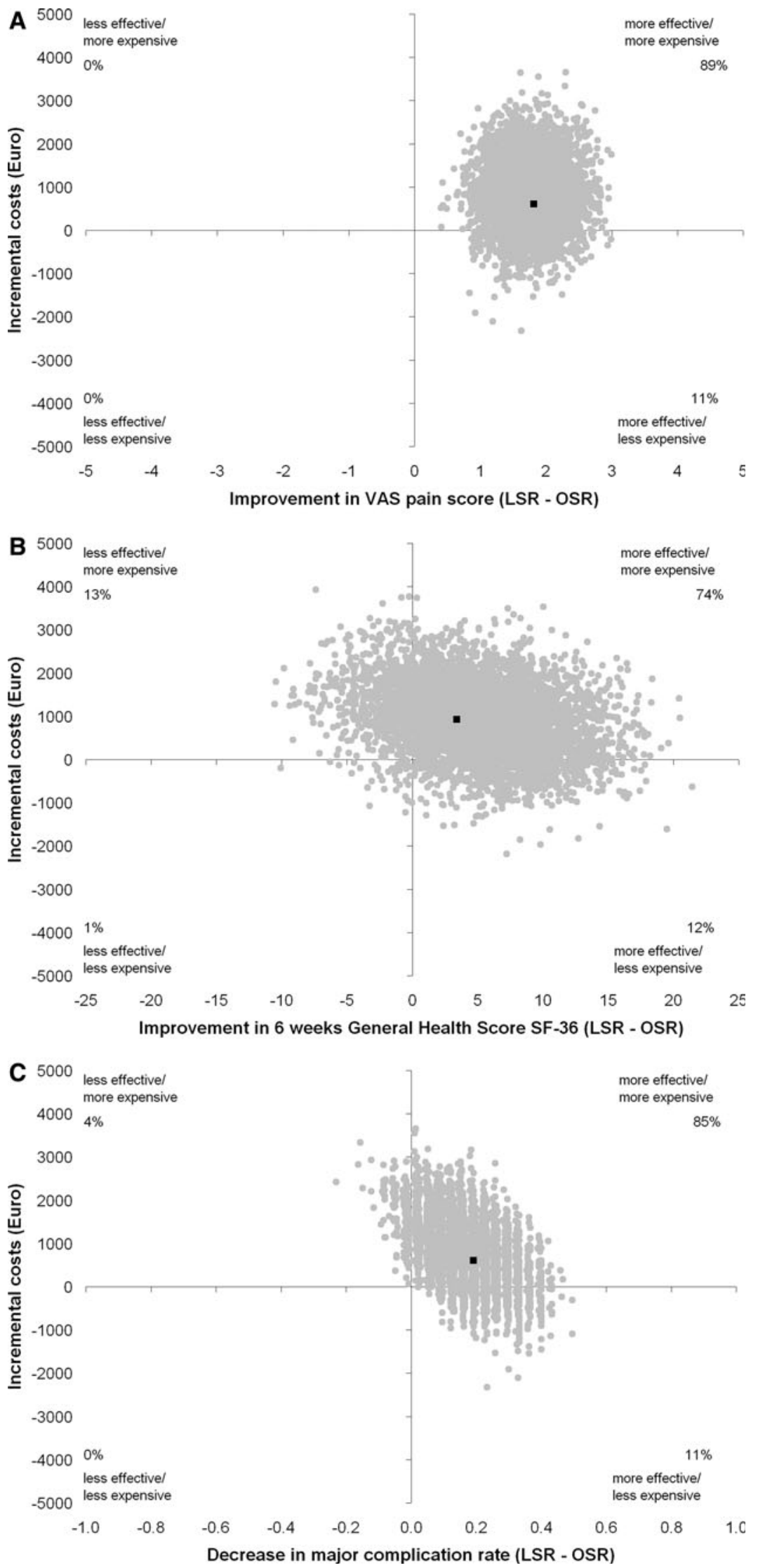
breakdown of the costs measured make these results difficult to compare to the current study. In the current study, cost-effectiveness ratios are presented for the VAS pain score, the general health score of the Short Form-36, and major complication rates, the latter being the primary end point of the Sigma trial. Pain and quality of life were chosen as effect measures for being the main determinants of subjective well-being of surgical patients [23, 24].

A limitation of this study may be the selection of patients from the VU University Medical Center. Only 57 patients of the total of 104 included in the multicenter Sigma trial were subjected to these cost analyses. Since no uniform or transparent cost registration exists for different hospitals in The Netherlands, let alone for various countries, cost-effectiveness analyses in this manner could not be performed. Moreover, problems arise in making international comparisons of costs because prices of resources may differ between countries. Publications on resource use and costs of laparoscopic versus open colorectal surgery report a wide spectrum of mean costs, ranging from $€ 1752$ [25] and $€ 3666$ [26] to $€ 20,380$ [27] and $€ 25,277$ [17]. These large differences reflect the heterogeneity of costs and their registration methods between hospitals and countries. Nevertheless, in a systematic review article, Dowson et al. [15] concluded that total hospital costs are similar for laparoscopic and open colorectal surgery. Therefore, cost should not be a deterrent to performing laparoscopic colorectal surgery.

In conclusion, total healthcare costs of laparoscopic and open elective sigmoid resections for symptomatic diverticular disease are similar. As the differences in clinical outcomes between the two treatment groups are in favor of the LSR group, candidates for an elective sigmoid resection should preferably be approached laparoscopically.

Acknowledgment We thank Meike van Haastert and Koen Meijssen from the Department of Information and Management for their contribution to the collection of resource utilization and the translation into costs.

Disclosures B. R. Klarenbeek, V. M. H. Coupé, D. L. van der Peet, and M. A. Cuesta have no conflicts of interest or financial ties to disclose.

\section{References}

1. Parks TG (1969) Natural history of diverticular disease of the colon. A review of 521 cases. Br Med J 4:639-642

2. Chapman JR, Dozois EJ, Wolff BG, Gullerud RE, Larson DR (2006) Diverticulitis: a progressive disease? Do multiple recurrences predict less favorable outcomes? Ann Surg 243:876-830

3. Sandler RS, Everhart JE, Donowitz M, Adams E, Cronin K, Goodman C, Gemmen E, Shah S, Avdic A, Rubin R (2002) The burden of selected digestive diseases in the United States. Gastroenterology 122:1500-1511

4. Etzioni DA, Mack TM, Beart RW Jr, Kaiser AM (2009) Diverticulitis in the United States: 1998-2005: changing patterns of disease and treatment. Ann Surg 249:210-217

5. Jacobs DO (2007) Clinical practice. Diverticulitis. N Engl J Med 357:2057-2066

6. Rafferty J, Shellito P, Hyman NH, Buie WD (2006) Practice parameters for sigmoid diverticulitis. Dis Colon Rectum 49:939-944

7. Janes S, Meagher A, Frizelle FA (2005) Elective surgery after acute diverticulitis. Br J Surg 92:133-142

8. Hjern F, Josephson T, Altman D, Holmstrom B, Johansson C (2008) Outcome of younger patients with acute diverticulitis. Br J Surg 95:758-764

9. Morris CR, Harvey IM, Stebbings WS, Hart AR (2008) Incidence of perforated diverticulitis and risk factors for death in a UK population. Br J Surg 95:876-881

10. Alves A, Panis Y, Slim K, Heyd B, Kwiatkowski F, Mantion G (2005) French multicentre prospective observational study of laparoscopic versus open colectomy for sigmoid diverticular disease. Br J Surg 92:1520-1525

11. Schwandner O, Farke S, Fischer F, Eckmann C, Schiedeck TH, Bruch HP (2004) Laparoscopic colectomy for recurrent and complicated diverticulitis: a prospective study of 396 patients. Langenbecks Arch Surg 389:97-103

12. Scheidbach H, Schneider C, Rose J, Konradt J, Gross E, Barlehner E, Pross M, Schmidt U, Kockerling F, Lippert H (2004) Laparoscopic approach to treatment of sigmoid diverticulitis: changes in the spectrum of indications and results of a prospective, multicenter study on 1,545 patients. Dis Colon Rectum 47:1883-1888

13. Klarenbeek BR, Veenhof AA, de Lange ES, Bemelman WA, Bergamaschi R, Heres P, Lacy AM, van den Broek WT, van der Peet DL, Cuesta MA (2007) The Sigma-trial protocol: a prospective double-blind multi-centre comparison of laparoscopic versus open elective sigmoid resection in patients with symptomatic diverticulitis. BMC Surg 7:16

14. Klarenbeek BR, Veenhof AA, Bergamaschi R, van der Peet DL, van den Broek WT, de Lange ES, Bemelman WA, Heres P, Lacy AM, Engel AF, Cuesta MA (2009) Laparoscopic sigmoid resection for diverticulitis decreases major morbidity rates: a randomized control trial: short-term results of the Sigma Trial. Ann Surg 249:39-44

15. Dowson HM, Huang A, Soon Y, Gage H, Lovell DP, Rockall TA (2007) Systematic review of the costs of laparoscopic colorectal surgery. Dis Colon Rectum 50:908-919

16. Senagore AJ, Duepree HJ, Delaney CP, Dissanaike S, Brady KM, Fazio VW (2002) Cost structure of laparoscopic and open sigmoid colectomy for diverticular disease: similarities and differences. Dis Colon Rectum 45:485-490

17. Liberman MA, Phillips EH, Carroll BJ, Fallas M, Rosenthal R (1996) Laparoscopic colectomy vs. traditional colectomy for diverticulitis. Outcome and costs. Surg Endosc 10:15-18

18. Kohler L, Rixen D, Troidl H (1998) Laparoscopic colorectal resection for diverticulitis. Int J Colorectal Dis 13:43-47

19. Barber JA, Thompson SG (2000) Analysis of cost data in randomized trials: an application of the non-parametric bootstrap. Stat Med 19:3219-3236

20. Thompson SG, Barber JA (2000) How should cost data in pragmatic randomised trials be analysed? BMJ 320:1197-1200

21. Briggs AH, Wonderling DE, Mooney CZ (1997) Pulling costeffectiveness analysis up by its bootstraps: a non-parametric approach to confidence interval estimation. Health Econ $6: 327-340$ 
22. Briggs A, Fenn P (1998) Confidence intervals or surfaces? Uncertainty on the cost-effectiveness plane. Health Econ 7:723-740

23. Coupé VM, Veenhof C, van Tulder MW, Dekker J, Bijlsma JW, van den Ende $\mathrm{CH}$ (2007) The cost effectiveness of behavioural graded activity in patients with osteoarthritis of hip and/or knee. Ann Rheum Dis 66:215-221

24. Aaronson NK, Muller M, Cohen PD, Essink-Bot ML, Fekkes M, Sanderman R, Sprangers MA, te Velde A, Verrips E (1998) Translation, validation, and norming of the Dutch language version of the SF-36 Health Survey in community and chronic disease populations. J Clin Epidemiol 51:1055-1068
25. Duepree HJ, Senagore AJ, Delaney CP, Brady KM, Fazio VW (2002) Advantages of laparoscopic resection for ileocecal Crohn's disease. Dis Colon Rectum 45:605-610

26. Kohler L, Holthausen U, Troidl H (1997) Laparoscopic colorectal surgery-attempt at evaluating a new technology. Chirurg 68:794-800

27. Pfeifer J, Wexner SD, Reissman P, Bernstein M, Nogueras JJ, Singh S, Weiss E (1995) Laparoscopic vs open colon surgery. Costs and outcome. Surg Endosc 9:1322-1326 